\title{
Nanofabrication Limits in Layered Ferroelectric Semiconductors via He-ion Beam
}

Holland Hysmith, ${ }^{1}$ Alex Belianinov ${ }^{1,2}$ Matthew J. Burch, ${ }^{1,2}$ Anton V. Ievlev, ${ }^{1,2}$ Vighter Iberi, ${ }^{1,3}$ Michael A. Susner, ${ }^{4}$ Michael A. McGuire, ${ }^{4}$ Peter Maksymovych,,${ }^{1,2}$ Marius Chyasnavichyus, ${ }^{1,2}$ Stephen Jesse, ${ }^{1,2}$ Olga S. Ovchinnikova ${ }^{1,2^{*}}$

1. Center for Nanophase Materials Sciences, Oak Ridge National Laboratory, Oak Ridge, TN 37831

2. The Institute for Functional Imaging of Materials, Oak Ridge National Laboratory, Oak Ridge, TN 37831

3. Department of Materials Science and Engineering, University of Tennessee, Knoxville, Knoxville TN 37996

4. Materials Sciences and Technology Division, Oak Ridge National Laboratory, Oak Ridge, TN 37831

Advances in nanofabrication are the driving force behind the technological breakthroughs in device performance and miniaturization. Functional layered materials with dielectric properties, such as insulators, semiconductors, and correlated electron materials will be necessary for fabrication and delivery of new age devices. ${ }^{1}$ Helium (and Neon) ion beam nanofabrication has the benefits of clean, resist-free processing; smaller penetration depth into the material, and reduced active ion implantation, common in standard Focused Ion Beam (FIB) lithography applications. ${ }^{2}$

We demonstrate the use of HIM to create a variety of micro-sized structures with varying chemical properties atop several transition metal thiophosphates ${ }^{3}$ (TPS) compositions; a broad class of van-derWaals layered solids. TPS compounds exhibit large band-gaps and a pronounced flexibility to ionic substitution in the metal sites with over 260 combinations known for the sulfides alone.

To investigate the effects of the stoichiometric ratio of $\mathrm{Cu} / \mathrm{In}$ on the growth of nanostructures, we irradiated the surface of the different $\mathrm{Cu}$ compositions with varying He doses. Figure 1 illustrates Band Excitation Piezoresponse Force Microscopy (BE-PFM) results for same levels of irradiation in $\mathrm{Cu}_{0.05} \mathrm{In}_{1.32} \mathrm{P}_{2} \mathrm{~S}_{6}$ and $\mathrm{Cu}_{0.7} \mathrm{In}_{1.1} \mathrm{P}_{2} \mathrm{~S}_{6}$. Irradiated areas with ferrielectrically active $\mathrm{Cu}$ phase grow at a higher rate vertically and horizontally as opposed to the paraelectric $\operatorname{In}_{4 / 3} \mathrm{P}_{2} \mathrm{~S}_{6}$. The nanostructures have shown an overall increase of area, volume. Our Time of Flight Secondary Ion Mass Spectrometry (ToF-SIMS) results, shown in Figure 2, show a significant increase in the amount of oxygen penetration into the sample as a function of dose.

In summary, we have explored the effect of helium ion impact on the surface of a library of TPS compounds using AFM and ToF-SIMS. After the helium exposure, we have used the AFM to observe surface nano- and micro- structures that scale, in area and volume, to the total dose of the helium ion beam, as well as the overall copper concentration in the sample. Furthermore, our ToF-SIMS results show that after ion irradiation, the oxygen concentration in the irradiated areas is significantly increased.

\section{References:}

[1] Chhowalla, M. et al. The chemistry of two-dimensional layered transition metal dichalcogenide nanosheets. Nature chemistry 5, 263-275 (2013). 
[2] David C Joy, Helium Ion Microscopy: Principles and Applications, First ed. (Springer, New York USA, Heidelberg Germany, Dordrecht Netherlands, London United Kingdom, 2013). [5] F Author in "Introduction to abstract writing", ed. D Writers, (Publisher, City) p.1.

[3] Belianinov, A. et al. Polarization control via He-ion beam induced nanofabrication in layered ferroelectric semiconductors. ACS applied materials \& interfaces 8, 7349-7355 (2016).

[4] Research was supported (M.B., H.H., V. I., A.V.I., M.C., P.M.,O. S. O) and partially conducted (AFM, HIM, ToF-SIMS) at the Center for Nanophase Materials Sciences, which is sponsored at Oak Ridge National Laboratory by the Scientific User Facilities Division, Office of Basic Energy Sciences, US Department of Energy. Research was partially sponsored by the Laboratory Directed Research and Development Program of Oak Ridge National Laboratory, managed by UT-Battelle, LLC, for the U. S. Department of Energy (HIM, A. B., crystal growth, M.A.S. and M.A.M.).

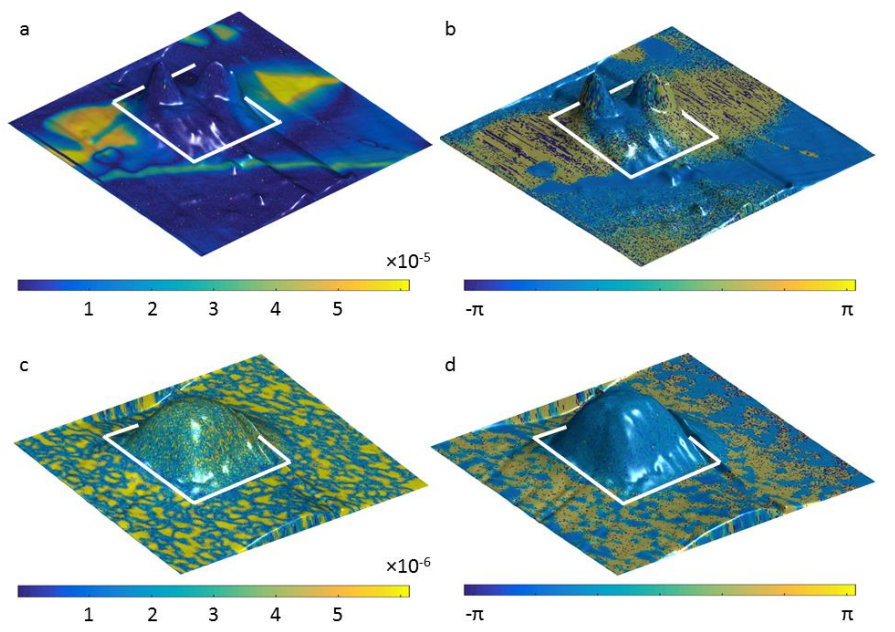

Figure 1: Band Excitation Piezoresponse Force Microscopy (BE-PFM) results. The BE-PFM amplitude $(\mathrm{a}, \mathrm{b})$ and phase $(\mathrm{c}, \mathrm{d})$ of $\mathrm{Cu}_{0.19}$ samples $(\mathrm{a}, \mathrm{c})$ and $\mathrm{Cu}_{0.70}(\mathrm{~b}, \mathrm{~d})$. The BE-PFM signal colormap for both samples is overlaid on the topography as captured by AFM. Black boxes in all images indicate the HIM irradiated areas.
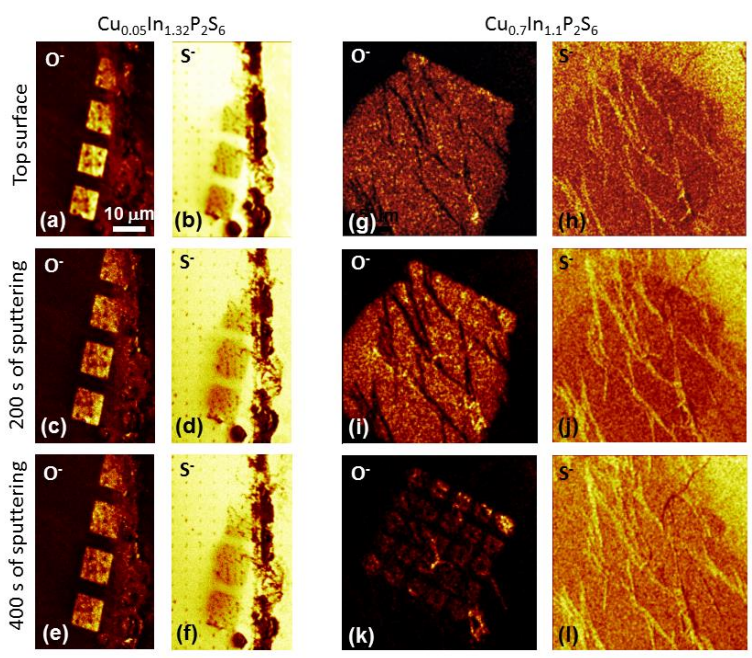

Figure 2: Time of Flight Secondary Ion Mass Spectrometry (ToF-SIMS) results on irradiated regions. Secondary ion mass spectrometry of oxygen of irradiated boxes in a (a-f) low $\mathrm{Cu}$ concentration sample $\left(\mathrm{Cu}_{0.05}\right)$ and a $(\mathrm{g}-\mathrm{l})$ high $\mathrm{Cu}$ concentration sample $\left(\mathrm{Cu}_{0.70}\right)$ at different sputtering times (depth) for both oxygen (a, c, e, g, i, k) and sulfur (b, d, f, h, j, l). 